\title{
A cohort study to identify risk factors for Plasmodium falciparum infection in Burkinabe children: implications for other high burden high impact countries
}

Jean Baptiste Yaro 1,2, Alphonse Ouedraogo ', Z. Amidou Ouedraogo ', Amidou Diarra', Malik Lankouande ', Efundem Agboraw ${ }^{3}$, Eve Worrall ${ }^{3}$, Kobié Hyacinthe Toe ${ }^{1}$, Antoine Sanou 1,4, W. Moussa Guelbeogo ${ }^{1}$, N'Fale Sagnon ${ }^{1}$, Hilary Ranson ${ }^{3}$, Alfred B. Tiono ${ }^{1}$, Steven W. Lindsay ${ }^{2}$ and Anne L. Wilson ${ }^{3 *}$

\begin{abstract}
Background: Progress in controlling malaria has stalled in recent years. Today the malaria burden is increasingly concentrated in a few countries, including Burkina Faso, where malaria is not declining. A cohort study was conducted to identify risk factors for malaria infection in children in southwest Burkina Faso, an area with high insecticide-treated net (ITN) coverage and insecticide-resistant vectors.

Methods: Incidence of Plasmodium falciparum infection was measured in 252 children aged 5 to 15 years, using active and passive detection, during the 2017 transmission season, following clearance of infection. Demographic, socio-economic, environmental, and entomological risk factors, including use of ITNs and insecticide resistance were monitored.
\end{abstract}

Results: During the six-month follow-up period, the overall incidence of $P$. falciparum infection was 2.78 episodes per child ( $95 \% \mathrm{Cl}=2.66-2.91$ ) by microscopy, and 3.11 ( $95 \% \mathrm{Cl}=2.95-3.28$ ) by polymerase chain reaction (PCR). The entomological inoculation rate (EIR) was 80.4 infective bites per child over the six-month malaria transmission season. At baseline, $80.6 \%$ of children were reported as sleeping under an ITN the previous night, although at the last survey, $23.3 \%$ of nets were in poor condition and considered no longer protective. No association was found between the rate of $P$. falciparum infection and either EIR (incidence rate ratio (IRR): $1.00,95 \% \mathrm{Cl}: 1.00-1.00, p=0.08$ ) or mortality in WHO tube tests when vectors were exposed to $0.05 \%$ deltamethrin (IRR: 1.05 , 95\% Cl: $0.73-1.50, p=0.79$ ). Travel history (IRR: $1.52,95 \% \mathrm{Cl}: 1.45-1.59, p<0.001$ ) and higher socio-economic status were associated with an increased risk of P. falciparum infection (IRR: 1.05, 95\% Cl: 1.00-1.11, $p=0.04$ ).

Conclusions: Incidence of $P$. falciparum infection remains overwhelmingly high in the study area. The study findings suggest that because of the exceptionally high levels of malaria transmission in the study area, malaria elimination cannot be achieved solely by mass deployment of ITNs and additional control measures are needed.

Keywords: Malaria, Epidemiology, Cohort study, Burkina faso, Vector control, Insecticide resistance

*Correspondence: Anne.wilson@lstmed.ac.uk

${ }^{3}$ Department of Vector Biology, Liverpool School of Tropical Medicine, Liverpool, UK

Full list of author information is available at the end of the article

\section{Background}

Malaria remains an acute public health problem throughout sub-Saharan Africa (SSA) with an estimated 213 million cases and 380,000 deaths in 2018

(c) The Author(s) 2020. This article is licensed under a Creative Commons Attribution 4.0 International License, which permits use, sharing, adaptation, distribution and reproduction in any medium or format, as long as you give appropriate credit to the original author(s) and the source, provide a link to the Creative Commons licence, and indicate if changes were made. The images or other third party material in this article are included in the article's Creative Commons licence, unless indicated otherwise in a credit line to the material. If material is not included in the article's Creative Commons licence and your intended use is not permitted by statutory regulation or exceeds the permitted use, you will need to obtain permission directly from the copyright holder. To view a copy of this licence, visit http://creativeco mmons.org/licenses/by/4.0/. The Creative Commons Public Domain Dedication waiver (http://creativecommons.org/publicdomain/ zero/1.0/) applies to the data made available in this article, unless otherwise stated in a credit line to the data. 
[1]. Despite unprecedented declines in malaria between 2000 and 2015 , of which $68 \%$ can be attributed to the scale-up of insecticide-treated nets (ITNs) [2], recent years have seen stagnating progress in high burden countries [1]. Reasons for this lack of progress are unclear, but may include incomplete coverage of ITNs, nets in poor condition and malaria vectors resistant to the pyrethroid insecticides used for ITNs. Burkina Faso, along with 10 other high-burden countries in Africa, plus India, has been designated as a High Burden to High Impact country by the World Health Organization (WHO) and the Roll Back Malaria Partnership which calls for an aggressive new approach to accelerate malaria control [3]. Burkina Faso has the seventh highest number of malaria cases globally [1], and has seen a rise in the number of malaria cases from 9 million in 2016 to over 11 million in 2017 and 2018 [4-6].

Despite the known protective efficacy of ITNs, the 2010 ITN universal coverage campaign in Burkina Faso did not decrease malaria and all-cause mortality in children younger than 5 years old, even with $92 \%$ of children reportedly sleeping under ITNs [7]. The study site in Burkina Faso has extremely high prevalence and intensity of pyrethroid resistance in malaria vectors $[8,9]$. For example, mosquitoes reared from larvae collected in Tengrela village between 2016 and 2018, showed only $2 \%$ mortality when exposed to the standard diagnostic dose of deltamethrin of $0.05 \%$ designed to kill all mosquitoes [10]. The public health impact of insecticide resistance, however, remains contested [11-14]. Insecticide resistance is only one important factor affecting the efficacy of nets, since ITN coverage, durability and whether people actually sleep under nets, as well as mosquito biting time and behaviour are also important. There is increasing evidence that socalled long-lasting insecticidal nets are not effective for 3 years [15-17], as claimed, and is the reason the term ITN is used. Coverage and system inefficiencies mean that ITNs are unevenly distributed among households and an estimated $50 \%$ of ITNs are lost from households after 23 months in Africa [18]. Social and environmental factors, including access to healthcare, socio-economic status (SES) and house construction, found to be protective against malaria in other studies [19], may also impact malaria risk in Burkina Faso.

A cohort study of children was conducted to determine risk factors for Plasmodium falciparum infection in an area of persistent and intense malaria transmission in rural Burkina Faso, with high ITN coverage and high levels of insecticide resistance. The study findings may help to identify potential opportunities for improving malaria control in Burkina Faso and other countries in SSA experiencing persistently high malaria transmission.

\section{Methods \\ Study site}

The study was conducted from June to December 2017 in Banfora Health District, Cascades region, southwest Burkina Faso (Fig. 1), an area of Sudanian savannah covering $6,295 \mathrm{sq} \mathrm{km}$ and with an estimated population of 407,073 inhabitants [4]. Subsistence farming and animal husbandry are the main activities. Banfora District has intense seasonal malaria transmission over a six-month period following seasonal rains from May to November [20]. Plasmodium falciparum accounts for $90 \%$ of cases [20]. The main malaria vector is Anopheles gambiae sensu stricto (s.s.), but Anopheles coluzzii is also found [21]. A universal coverage (defined as one ITN for every two persons at risk of malaria) campaign in 2016 distributed ITNs with permethrin or deltamethrin (Sumitomo Chemical, Vestergaard and BASF) and therefore no new ITNs were distributed by the study. No indoor residual spraying (IRS) was conducted. Since 2014, children under 5 years of age in the study area receive seasonal malaria chemoprevention (SMC) during the transmission season [22].

\section{Recruitment of study cohort}

Ten villages were randomly selected from a list of villages in the study area following a two-stage process. Firstly, an area spanning the catchment areas of five health centres was chosen. Each health centre had a catchment radius of approximately $10 \mathrm{~km}$. Secondly, two villages at least $3 \mathrm{~km}$ apart were chosen at convenience from each catchment area, giving a total of 10 villages. Thirty children were randomly selected from each village using the Health and Demographic Surveillance System enumeration list. Children were eligible to participate if they were aged 5-15 years, likely to remain resident in the village for the duration of the study and the caregiver provided informed consent (assent of child if aged 12-15 years). Older children were selected as they have the highest malaria incidence, relatively low immunity and contribute substantially to transmission [23], while children under 5 years were excluded due to roll-out of SMC. Children were not eligible to participate if they were taking part in a malaria clinical trial, or had a contra-indication to the artemisinin-combination therapy (ACT), artemether-lumefantrine (AL). At enrolment in June 2017, 300 children provided a blood film. Irrespective of their malaria parasite status, all children received a curative dose of AL (Wellona Pharma Private Limited, Nana Varachha, Surat, India) to clear any existing parasitaemia. Children were revisited 21 days later, at which time two 


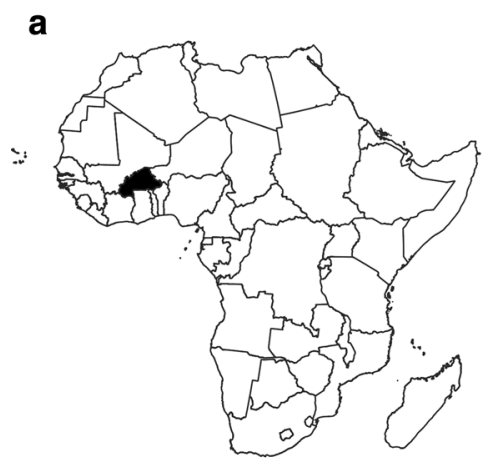

b

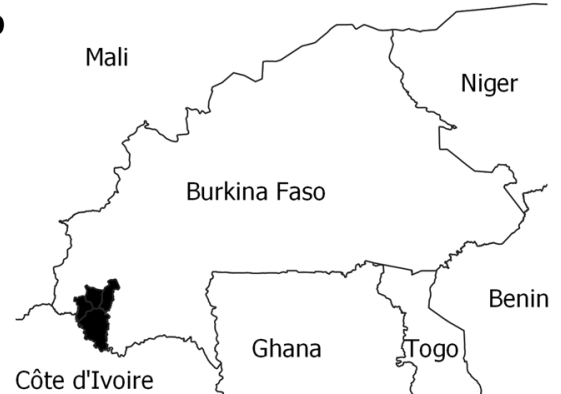

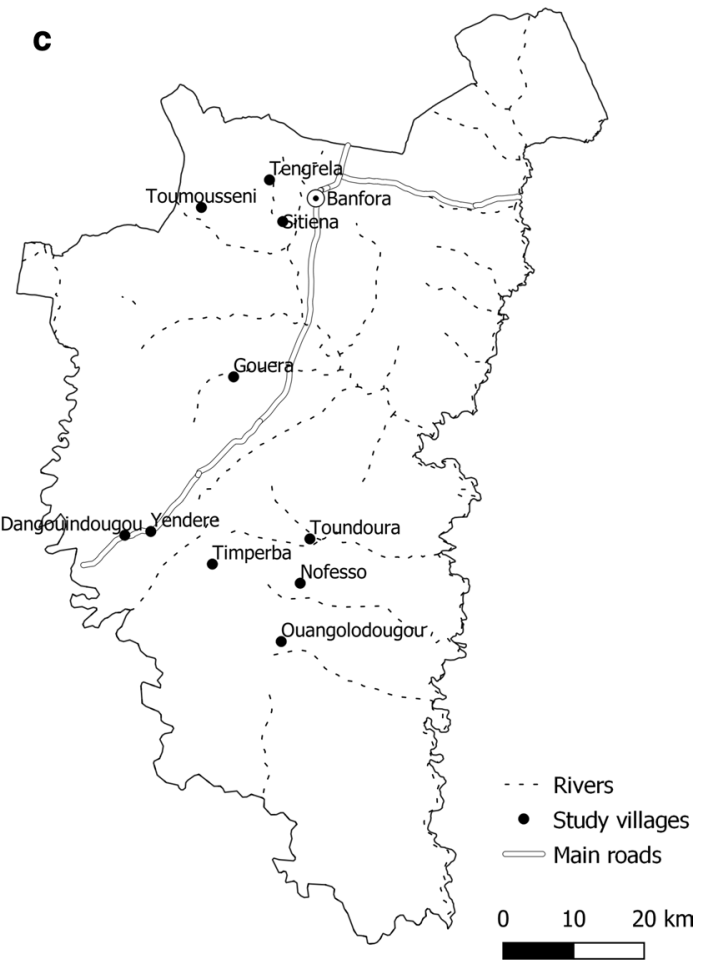

Fig. 1 Study location. a Location of Burkina Faso; b Location of study site in Burkina Faso; c Location of the 10 study villages and Banfora (provincial capital) in study site

blood slides and a blood spot were taken and examined to ensure parasite clearance. Those with a negative parasite status confirmed by polymerase chain reaction (PCR) were enrolled in the study. Children re-infected in the 21 day period were re-treated with $\mathrm{AL}$ and were eligible for enrolment in the study after 28 days once parasite clearance was confirmed using PCR.

\section{Follow-up of study cohort}

Symptomatic and asymptomatic Plasmodium infections were recorded using both active and passive detection. Study children were visited at home every 2 weeks by fieldworkers during the peak transmission season, from July-December 2017. At each visit, fieldworkers measured a child's axillary temperature and prepared two blood films and a filter paper blood spot. Children with an axillary temperature $\geq 37.5{ }^{\circ} \mathrm{C}$ or history of fever in the previous $48 \mathrm{~h}$ were advised to visit the local health centre and, if they were a malaria case, treated with AL, following National Malaria Control Programme (NMCP) guidelines [24]. If a child was absent at the time of the visit, the fieldworker made one more attempt to locate them the following day, after which the child was recorded as being absent. Caregivers were encouraged by the study team at enrolment and at fortnightly visits to take their child to the nearest government health centre should the child have a fever or feel unwell. Travel and treatment costs for sick children were reimbursed by the study. Study nurses were posted in the five health centres close to the study villages and performed rapid diagnostic tests for malaria (SD BIOLINE Malaria Ag P.f/Pan, Abbott Laboratories, Illinois, USA) in study children presenting with an axillary temperature $\geq 37.5^{\circ} \mathrm{C}$ or history of fever within the previous $48 \mathrm{~h}$, and prepared two blood films and a filter paper blood spot. Children diagnosed with clinical malaria received AL [24]. Clinical data were recorded on dedicated study logs and transcribed by fieldworkers who visited each nurse weekly.

Thick blood films were stained with Giemsa and examined under 1000-fold magnification by experienced microscopists at the Centre National de Recherche et de Formation sur le Paludisme (CNRFP) in Banfora. Parasite counts were recorded per high power field and 100 fields counted before a slide was declared negative. Two blood slides from each subject were read separately by two microscopists. Discrepancies in positive and negative reads and parasite counts differing by more than tenfold between the two reads were resolved by the supervisor. Filter paper blood samples were analysed for the presence of 18 s rRNA gene using PCR [25]. 


\section{Risk factors}

At enrolment, sociodemographic characteristics of the child (age, gender, religion, ethnicity) and their caregiver (education, occupation) were recorded. Questionnaires recorded the presence or absence of large domestic animals (cattle, goats, sheep, donkeys, horses) within $5 \mathrm{~m}$ of each study child's house and the materials used to construct the building in which the study child slept, including presence of a metal roof, eaves and door and window screening. The head of household completed a questionnaire on their asset ownership, house construction and other variables, following standard procedures used in the Burkina Faso Malaria Indicator Survey (MIS) [26]. Houses of study children were geolocated using a global positioning system (GARMIN eTrex 20).

Use of an ITN the previous night by each study child and use of spatial or topical repellents were recorded at enrolment and each active visit. Survivorship and integrity of each study child's ITN was measured in July, October and December. Each net was recorded as being in use (i.e., hanging over the study child's sleeping space), in storage, being washed, or lost. Loss was categorized as: (i) net given away voluntarily; (ii) net stolen; or, (iii) net destroyed, discarded or used for alternative purposes. Fabric integrity of the net was assessed by counting the number of holes and their size according to WHO guidance [27]. A weighted sum of hole counts, the proportionate hole index (pHI), was calculated with a pHI of 0-64 categorized as good, 65-642 as acceptable and $643+$ as too torn and non-protective [28]. To measure ITN bio-efficacy, 26 bed nets were sampled (at least 2 randomly selected nets per village, except for Sitiena village where 1 net was tested) in October 2017 and stored at $+4{ }^{\circ} \mathrm{C}$. ITNs taken for testing were replaced with new ones. WHO cone bioassays were performed using the pyrethroid-susceptible Kisumu strain of An. gambiae sensu lato (s.l.) at the CNRFP insectaries in Banfora using the WHO efficacy requirement of $\geq 80 \%$ mortality [29].

Mosquitoes were sampled with CDC light traps, positioned with the light $1 \mathrm{~m}$ above the ground at the foot end of the bed of each study child sleeping under an ITN from 19.00 to 06.00 , every 4 weeks from July to December 2017. In addition, each child's net was systematically searched for mosquitoes between 06.00 and 07.00 every 4 weeks using a torch. Mosquitoes were identified morphologically using established keys and female $A n$. gambiae s.l. typed to species using PCR. The presence of sporozoites in An. gambiae s.l. was determined using an enzyme-linked immunosorbent assay [30]. Larval surveys to determine the proximity of anopheline larval habitats to study children's houses were carried out in the vicinity of all 10 villages during September 2017. All types of larval habitats were surveyed including irrigated fields, puddles, muddy foot or hoof prints, streams and ponds.

Phenotypic insecticide resistance was measured using WHO tube tests as per standard procedures [31]. Assays were performed with An. gambiae s.l. mosquitoes reared from immatures collected in seven study villages (larvae were not found in the other three villages).

\section{Data management and statistical analysis}

Data were collected on android personal digital assistants programmed using KoboCollect and included dropdown boxes and consistency checks to reduce data entry errors. Following cleaning, the dataset was locked and saved in Microsoft Access. An analytical plan was developed prior to data analysis.

The primary outcome was the incidence rate of microscopically confirmed $P$. falciparum infection during the transmission season, detected using active and passive case detection. PCR-confirmed P. falciparum incidence rate was a secondary outcome. After ACT treatment, further infections were censored for 28 days since infections during this time were most likely due to recrudescent parasites. Time at risk was also censored for time that study children spent away from their compound should they be found to be absent at the two-weekly home visits. The entomological inoculation rate (EIR) or estimated number of infectious bites per study child during the transmission season was calculated using the formula $\mathrm{EIR}=M a S d$ where $M a$ is the human biting rate, estimated from the arithmetic mean number of female An. gambiae s.l. caught per light trap night across the sixmonth transmission season, where $S$ is the proportion of female An. gambiae s.l. found to be sporozoite positive by village and $d$ is the number of days in the transmission season (n). Cone bioassay results for the netting pieces from each sampled net were pooled by village and by net type. QGIS Geographic Information System (QGIS Development Team (2019), Open Source Geospatial Foundation Project) was used to determine distances between the child's home and aquatic habitats. Distance to the nearest health facility was determined based on the shortest distance to travel by road. Principal component analysis was used to calculate a SES factor score based on asset ownership and household characteristics. SES factor scores (ranging from -1.8 to 3.2) were ranked and households divided into five equal wealth quintiles (1 poorest, through to 5 , least poor).

Mean values were compared using a t-test and proportions compared using Chi-squared tests. Poisson regression models were used to identify risk factors associated with $P$. falciparum infection incidence rate, adjusting for clustering by village. Risk factors assessed were: child's age, gender, ethnic group, religion, caregiver's education 
and occupation, wealth quintile and SES factor score, use of ITNs and other personal protection, ITN integrity, number of people sleeping in the room with the study child, housing materials (roof, eaves, wall and floor material, door and window screening), presence of cows, horses, sheep or goats within $5 \mathrm{~m}$ of the child's home, Euclidean distance to the nearest aquatic habitat with larval anophelines, distance by road to the nearest health centre, EIR at village level, and percentage mortality of An. gambiae s.l. mosquitoes from the village when exposed to $0.05 \%$ deltamethrin in a WHO tube test. A multivariate regression model was developed using a forward stepwise approach. Statistical analysis was carried out in Stata 15 (Statacorp, Texas, USA).

Assuming two P. falciparum infections per child during the transmission season, an intraclass correlation coefficient of 0.1 (design effect of 3.4), and a loss to follow-up of $10 \%$, the study had greater than $90 \%$ power to detect effect sizes of $>50 \%$ at the $5 \%$ level of significance, assuming $50 \%$ prevalence of the risk factor of interest in the population using the formula for comparison of two rates [32]. The study is reported following STROBE guidelines [33].

\section{Results}

Of 300 randomly selected children, 252 children aged 5-15 years old were enrolled in the cohort after confirmation of parasite clearance by PCR (Fig. 2). Of these 252 children, 228 were clear of infection at day 21 post-ACT, while an additional 24 children were enrolled following receipt of a second course of ACT due to re-infection. Three children withdrew from the study due to migration. No deaths were recorded among the cohort during the study period.

The mean age of cohort participants was 9.9 years, $52.0 \%$ of whom were male (Table 1 ). $38.9 \%$ of children were Gouin, 21.8\% Karaboro, 11.5\% Mossi, 9.1\% Turka, 6.3\% Fulani, and 4.4\% Senoufo. Caregivers were predominantly illiterate $(79.0 \%)$ and farmers (95.2\%). Most sleeping rooms of the children had metal roofs $(75.8 \%)$, brick walls $(57.9 \%)$ and cement floors (70.2\%). Over half the children's sleeping rooms had open eaves (54.8\%) and the vast majority did not have window screening (96.0\%).

During the follow-up period, 249 of the 252 children experienced at least one $P$. falciparum infection, as detected by microscopy. 31 children $(12.3 \%)$ had one $P$. falciparum infection, 139 (55.2\%) children experienced two P. falciparum infections, 75 (29.8\%) experienced three $P$. falciparum infections, and $4(1.6 \%)$ experienced

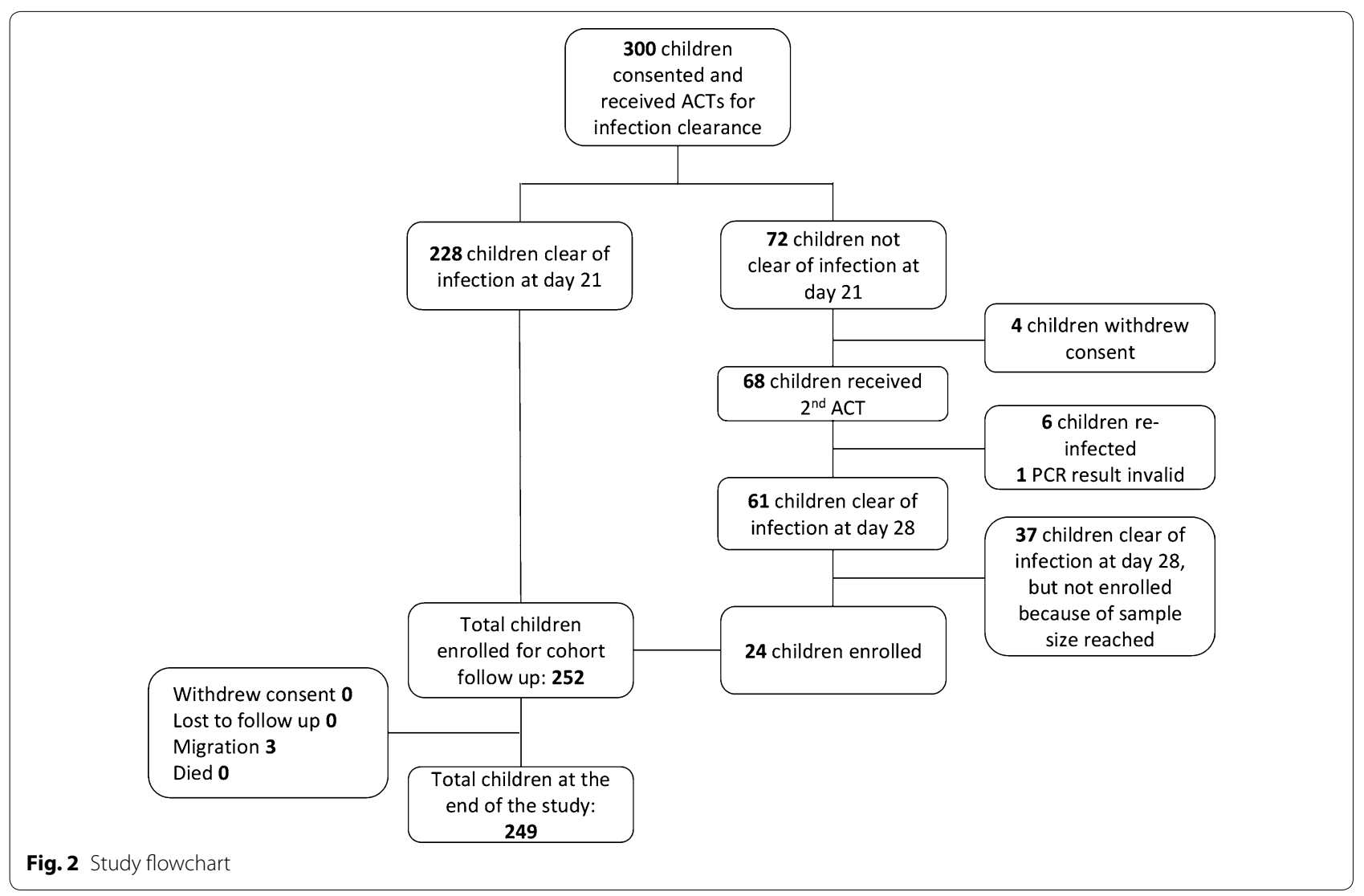


Table 1 Baseline characteristics of the study cohort

\begin{tabular}{|c|c|}
\hline Characteristic & Number (\%) $\mathrm{N}=252$ \\
\hline \multicolumn{2}{|l|}{ Age at enrolment } \\
\hline 5 years to $<8$ years & $76(30.2 \%)$ \\
\hline$\geq 8$ years & $176(69.8 \%)$ \\
\hline Age (mean/standard deviation) & $9.93(2.8)$ \\
\hline \multicolumn{2}{|l|}{ Gender } \\
\hline Male & $131(52.0 \%)$ \\
\hline Female & $121(48.0 \%)$ \\
\hline \multicolumn{2}{|l|}{ Ethnicity } \\
\hline Gouin & 98 (38.9\%) \\
\hline Karaboro & $55(21.8 \%)$ \\
\hline Mossi & $29(11.5 \%)$ \\
\hline Turka & $23(9.1 \%)$ \\
\hline Fulani & $16(6.3 \%)$ \\
\hline Senoufo & $11(4.4 \%)$ \\
\hline Others & $20(7.9 \%)$ \\
\hline \multicolumn{2}{|l|}{ Reported bed net use } \\
\hline Used bed net usually & $215(85.3 \%)$ \\
\hline Used a bed net the previous night & $203(80.6 \%)$ \\
\hline \multicolumn{2}{|l|}{ Caregiver's education level } \\
\hline Illiterate & $199(79.0 \%)$ \\
\hline Primary school & $45(17.9 \%)$ \\
\hline Secondary school or above & $8(3.2 \%)$ \\
\hline \multicolumn{2}{|l|}{ Caregiver's occupation } \\
\hline Not working/retired & $6(2.4 \%)$ \\
\hline Farmer & $240(95.2 \%)$ \\
\hline Commercial activities / government officer & $6(2.4 \%)$ \\
\hline \multicolumn{2}{|l|}{ Eave status of child's sleeping room } \\
\hline Closed & $102(40.5 \%)$ \\
\hline Open & $138(54.8 \%)$ \\
\hline \multicolumn{2}{|l|}{ Roof material of child's sleeping room } \\
\hline Metal & $191(75.8 \%)$ \\
\hline Thatch & $34(13.5 \%)$ \\
\hline Other roof type & $18(7.1 \%)$ \\
\hline \multicolumn{2}{|l|}{ Wall material of child's sleeping room } \\
\hline Mud & $65(25.8 \%)$ \\
\hline Brick & $146(57.9 \%)$ \\
\hline Cement (plastered or painted) & $32(12.7 \%)$ \\
\hline \multicolumn{2}{|l|}{ Floor material of child's sleeping room } \\
\hline Cement & $177(70.2 \%)$ \\
\hline Dirt floor & $65(25.8 \%)$ \\
\hline Tiles & $1(0.4 \%)$ \\
\hline \multicolumn{2}{|l|}{ Window screening of child's sleeping room } \\
\hline Absent & $242(96.0 \%)$ \\
\hline Present & $1(0.4 \%)$ \\
\hline
\end{tabular}

four $P$. falciparum infections. A total of 550 P. falciparum infections were identified using microscopy while 608 infections were identified using PCR. Of the $550 P$. falciparum infections confirmed using microscopy, 528
(96.0\%) were detected using active case detection and 22 (4.0\%) detected using passive case detection. Infections detected passively had a higher geometric mean $P$. falciparum density $(19,875 \mathrm{~mL}, 95 \% \mathrm{CI}=7896-31,854)$ compared to those detected through active surveillance (3744 $\mathrm{mL}, 95 \% \mathrm{CI}=2,691-4,797, \mathrm{p}<0.001)$. The $P$. falciparum infection incidence rate was 2.78 episodes per child during the six-month transmission season $(95 \%$ $\mathrm{CI}=2.66-2.91)$ by microscopy, and 3.11 episodes $(95 \%$ $\mathrm{CI}=2.95-3.28$ ) by PCR. Among children suffering from at least one infection, the median time to first infection detected by microscopy was 27 days (range 14-123 days).

At baseline, $80.6 \%$ of caregivers reported that the study child slept under an ITN the previous night. Reported ITN use the previous night at the baseline survey was greater than $82 \%$ across the four lowest SES quintiles compared to the least poor children (quintile $5=53.2 \%$, $p<0.001)$. Most study children's bed nets were either Permanet $2.0(52.0 \%)$ or Olyset net (16.7\%), with $85.5 \%$ provided by the NMCP and $4.0 \%$ purchased on the open market. Sleeping place inspections in July, October and December found $87.8 \%$ of children had a bed net hanging over their sleeping place (638 out of 727 observations). The most common reason for not having a net hanging was loss of the net (59/89 observations, $66.3 \%)$, while $15.7 \%$ (14/89 observations) of nets were stored and $2.2 \%$ (2/89 observations) being washed. The proportions lost, stored or being washed did not differ across the three surveys. Of the nets that were reported as being lost, $76.3 \%$ (45/59 observations) were destroyed, $22.0 \%$ (13/59 observations) stolen and 1.7\% (1/59 observations) given to friends or family. It was common for children to share a bed net with siblings and $61.4 \%$ of children slept with three or more children. At the last survey in December, $62.2 \%(155 / 249)$ of ITNs were in good condition, $14.5 \%$ (36/249) were damaged and $23.3 \%$ (58/249) badly torn and non-protective. Net condition did not differ significantly by survey round, although there was a tendency towards net deterioration over the study period. Cone testing of a random sample of 26 children's bed nets gave a mean knockdown at $1 \mathrm{~h}$ of $76.5 \%(95 \% \mathrm{CI}=61.9$ $91.1 \%)$ and mortality after $24 \mathrm{~h}$ of $42.2 \%(95 \% \mathrm{CI}=18.8$ $65.6 \%)$ for Olyset nets (9 nets, permethrin-treated) and mean knockdown of $85.2 \%(95 \% \mathrm{CI}=77.9-92.4 \%)$ and 24 h mortality of $39.9 \%$ (95\% CI $=27.7-52.0 \%)$ for Permanet (17 nets, deltamethrin-treated).

A total of 20,929 mosquitoes were caught from 1,151 trap collections with 16,270 of these being An. gambiae s.l. Highest densities of An. gambiae s.l. occurred in August (Fig. 3). Tengrela village had the highest density of An. gambiae s.l. across the season, reaching a peak of 137 An. gambiae s.l. per trap /night in August. Of the 7,615 identified to species, 4,101 (53.9\%) were An. gambiae s.s. 


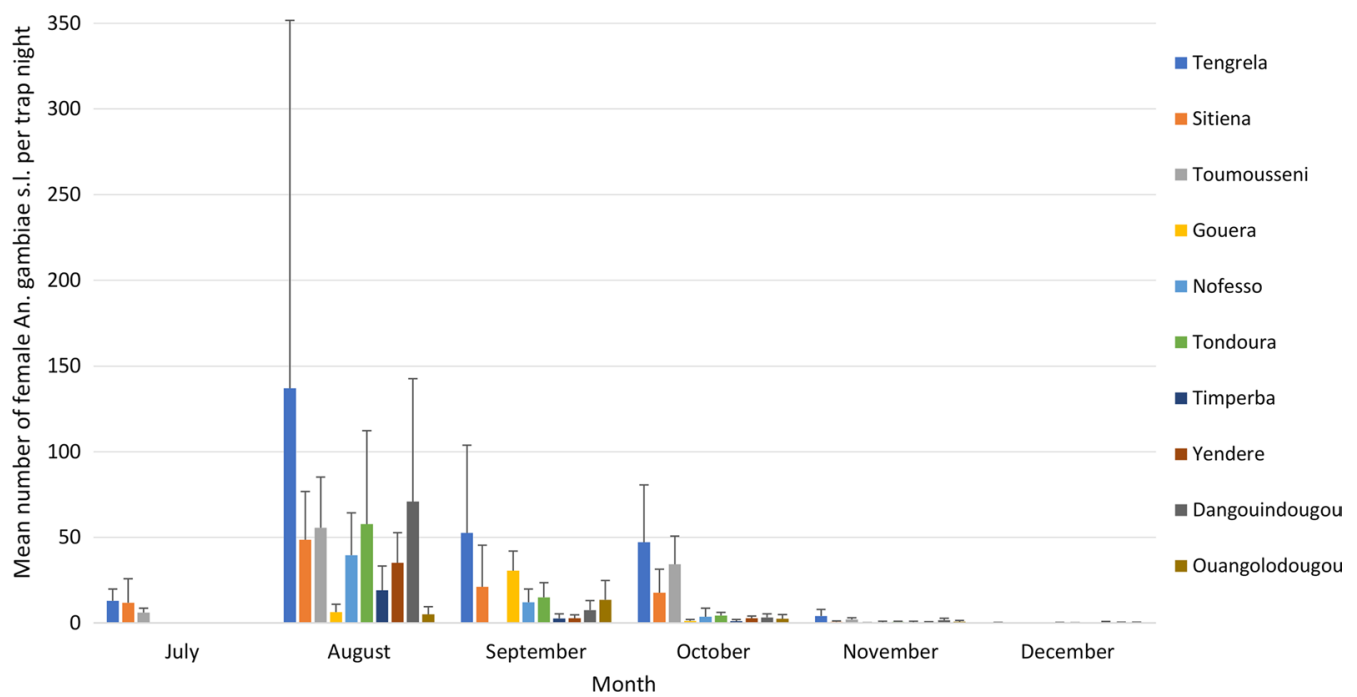

Fig. 3 Mean number of Anopheles gambiae s.l. per trap night in sleeping rooms of study children during the transmission season

and 2,590 (34.0\%) An. coluzzii. 3.3\% of the An. gambiae s.l. were sporozoite positive. The overall EIR in the study area was 80.4 infective bites/child over the six-month transmission season. The village-level EIR ranged from 40.8 in Timperba to 191.9 in Tondoura. Monthly systematic searches of study children's bed nets in the early morning did not collect any mosquitoes. Anopheles gambiae s.l. were resistant to $0.05 \%$ deltamethrin in WHO tube tests with a mean corrected mortality of $52.3 \%$ (95\% $\mathrm{CI}=26.4-78.2 \%$ ), with values ranging from $27.2 \%$ mortality in Toumousseni to $93.0 \%$ in Yendere.

The mean number of water bodies with anopheline larvae within $500 \mathrm{~m}$ of study houses was 8.7 (95\% CI $=7.0$ 10.3). The average distance by road from the study house to the nearest clinic was $3.6 \mathrm{~km}(95 \% \mathrm{CI}=3.2-4.0)$, although for the villages of Toundoura and Gouera, which did not have a clinic nearby, the distance was $7.5 \mathrm{~km}$ and $9.4 \mathrm{~km}$, respectively.

Univariate Poisson regression analysis identified two variables associated with incidence of $P$. falciparum infection in the study children and both remained significant in a multivariate model (Table 2). Although a small number of children travelled from the study area, this was associated with a $52 \%$ increase in the incidence of $P$. falciparum infection compared to children that did not travel (incidence rate ratio (IRR): 1.52, 95\% CI: 1.45-1.59, $p<0.001)$. The least poor children were found to have a higher incidence of $P$. falciparum infection. A 1 unit increase in the SES factor score was associated with a 5\% increase in infection rate (IRR: 1.05, 95\% CI: 1.00-1.11, $p=0.04)$.

\section{Discussion}

An extremely high incidence of $P$. falciparum infection was observed, with only three of the 252 cohort children remaining free from infection during the six-month follow-up period and $86.5 \%$ of children experiencing two or more infections. This high incidence of infection, despite regular retreatment with an effective anti-malarial if a child was infected, indicates the high force of infection in the study area and is supported by an estimated EIR of 80.4 infective bites per child during the transmission season. Similarly high levels of $P$. falciparum infection and malaria morbidity have been reported from other recent studies in Burkina Faso in areas of high ITN coverage [34-36].

The assessment of socio-demographic, entomological and environmental risk factors for P. falciparum infection did not elucidate any strong associations. This may be because of the overwhelming force of infection in the study area which meant that all the children were at extremely high risk of infection. Nevertheless, there was an indication of increased malaria risk in the few children that travelled overnight during the study period. Whether staying with family or for farming reasons, these children may be at higher risk of $P$. falciparum infection because they lack or have limited access to malaria prevention including ITNs, and diagnosis and treatment [37]. Overnight travel history was a risk factor for malaria in Uganda in a recent cohort study, with those not using ITNs at particularly high risk [38]. The finding of an association between higher SES factor score and a higher incidence of $P$. falciparum infection was unexpected since it is widely reported that the least poor children are typically at lower malaria risk than 
Table 2 Risk factors for Plasmodium infection incidence among children aged 5-15 years in Banfora region of Burkina Faso

\begin{tabular}{|c|c|c|c|c|c|c|c|c|}
\hline \multirow[t]{2}{*}{ Variables } & \multirow{2}{*}{$\begin{array}{l}\text { Number } \\
\text { of children }\end{array}$} & \multirow{2}{*}{$\begin{array}{l}\text { Number } \\
\text { of infections }\end{array}$} & \multirow{2}{*}{$\begin{array}{l}\text { Time } \\
\text { at risk } \\
\text { (years) }\end{array}$} & \multirow[t]{2}{*}{ Rate } & \multicolumn{2}{|c|}{ Univariate analysis } & \multicolumn{2}{|c|}{ Multivariate analysis } \\
\hline & & & & & IRR (95\% CI) & $P$ value & IRR $(95 \% \mathrm{Cl})$ & $P$ value \\
\hline \multicolumn{9}{|l|}{ Age (years) } \\
\hline $5-7$ & 76 & 164 & 31.09 & 5.28 & - & - & & \\
\hline $8-15$ & 176 & 386 & 71.40 & 5.41 & $1.02(0.94-1.12)$ & 0.59 & & \\
\hline \multicolumn{9}{|l|}{ Gender } \\
\hline Male & 131 & 288 & 52.93 & 5.44 & - & - & & \\
\hline Female & 121 & 262 & 49.56 & 5.29 & $0.97(0.90-1.05)$ & 0.46 & & \\
\hline \multicolumn{9}{|l|}{ Ethnic group } \\
\hline Other ethnicity & 236 & 519 & 95.82 & 5.42 & - & - & & \\
\hline Fulani & 16 & 31 & 6.66 & 4.65 & $0.86(0.65-1.13)$ & 0.28 & & \\
\hline \multicolumn{9}{|l|}{ Caregiver's education } \\
\hline Illiterate & 199 & 443 & 80.45 & 5.51 & - & - & & \\
\hline Literate & 53 & 107 & 22.04 & 4.86 & $0.88(0.76-1.02)$ & 0.09 & & \\
\hline \multicolumn{9}{|l|}{ Caregiver's occupation } \\
\hline Farmer & 240 & 523 & 97.39 & 5.37 & - & - & & \\
\hline Non farmer & 12 & 27 & 5.09 & 5.30 & $0.99(0.83-1.18)$ & 0.89 & & \\
\hline \multicolumn{9}{|l|}{ Religion } \\
\hline Muslims & 170 & 375 & 68.97 & 5.44 & - & - & & \\
\hline Christians & 33 & 67 & 13.93 & 4.81 & $0.88(0.77-1.02)$ & 0.09 & & \\
\hline Animists & 49 & 108 & 19.58 & 5.51 & $1.01(0.87-1.18)$ & 0.85 & & \\
\hline \multicolumn{9}{|l|}{ SES quintile } \\
\hline Poorest & 46 & 102 & 20.16 & 5.06 & $1.04(0.99-1.09)$ & 0.14 & & \\
\hline Poor & 44 & 95 & 18.47 & 5.14 & & & & \\
\hline Middle & 44 & 98 & 18.21 & 5.38 & & & & \\
\hline Rich & 46 & 103 & 19.34 & 5.33 & & & & \\
\hline Richest & 47 & 106 & 17.79 & 5.96 & & & & \\
\hline \multicolumn{9}{|l|}{ SES factor score } \\
\hline 1 unit increase & - & - & - & - & $1.06(1.01-1.11)$ & 0.03 & $1.05(1.00-1.11)^{*}$ & 0.04 \\
\hline \multicolumn{9}{|c|}{ Travel history during the study period } \\
\hline No & 246 & 537 & 100.93 & 5.32 & - & & & \\
\hline Yes & 6 & 13 & 1.55 & 8.39 & $1.58(1.31-1.90)$ & $<0.001$ & $1.52(1.45-1.59)^{\$}$ & $<0.001$ \\
\hline \multicolumn{9}{|c|}{ Slept under bed net previous night } \\
\hline Yes & 203 & 448 & 83.55 & 5.36 & - & - & & \\
\hline No & 49 & 102 & 18.93 & 5.39 & $1.00(0.78-1.29)$ & 0.97 & & \\
\hline \multicolumn{9}{|c|}{ Number of people sleeping in the child room (including child) } \\
\hline$\leq 6$ & 55 & 124 & 23.10 & 5.37 & - & & & \\
\hline $6<$ no. $\leq 12$ & 118 & 265 & 46.32 & 5.72 & $1.07(0.94-1.21)$ & 0.33 & & \\
\hline$>12$ & 79 & 161 & 33.06 & 4.87 & $0.91(0.79-1.04)$ & 0.16 & & \\
\hline \multicolumn{9}{|c|}{ Bed net integrity (at final survey in December) } \\
\hline Good (pHI: 0-64) & 155 & 331 & 62.63 & 5.28 & - & & & \\
\hline Damaged (pHI: 65-642) & 36 & 81 & 15.71 & 5.16 & $0.98(0.83-1.15)$ & 0.76 & & \\
\hline Too torn (pHI: $643+)$ & 58 & 134 & 23.52 & 5.70 & $1.08(0.97-1.20)$ & 0.18 & & \\
\hline \multicolumn{9}{|c|}{ Used other personal protective measures } \\
\hline No & 184 & 407 & 74.64 & 5.45 & - & - & & \\
\hline Yes & 58 & 125 & 24.53 & 5.10 & $0.93(0.81-1.08)$ & 0.36 & & \\
\hline Housing: roof of child's slee & & & & & & & & \\
\hline Metal & 191 & 420 & 77.60 & 5.41 & - & & & \\
\hline Non metal & 52 & 114 & 21.85 & 5.22 & $0.96(0.85-1.09)$ & 0.57 & & \\
\hline
\end{tabular}


Table 2 (continued)

\begin{tabular}{|c|c|c|c|c|c|c|c|c|}
\hline \multirow[t]{2}{*}{ Variables } & \multirow{2}{*}{$\begin{array}{l}\text { Number } \\
\text { of children }\end{array}$} & \multirow{2}{*}{$\begin{array}{l}\text { Number } \\
\text { of infections }\end{array}$} & \multirow{2}{*}{$\begin{array}{l}\text { Time } \\
\text { at risk } \\
\text { (years) }\end{array}$} & \multirow[t]{2}{*}{ Rate } & \multicolumn{2}{|c|}{ Univariate analysis } & \multicolumn{2}{|c|}{ Multivariate analysis } \\
\hline & & & & & IRR (95\% CI) & $P$ value & IRR (95\% CI) & $P$ value \\
\hline \multicolumn{9}{|c|}{ Housing: floor of the child's sleeping room } \\
\hline Cement/tiles & 178 & 388 & 72.91 & 5.32 & - & - & & \\
\hline Dirt & 65 & 146 & 26.54 & 5.50 & $1.03(0.94-1.13)$ & 0.48 & & \\
\hline \multicolumn{9}{|c|}{ Housing: wall of the child's sleeping room } \\
\hline Mud & 65 & 151 & 26.54 & 5.69 & - & - & & \\
\hline Brick & 146 & 309 & 59.15 & 5.22 & $0.92(0.83-1.02)$ & 0.12 & & \\
\hline Cement & 32 & 74 & 13.77 & 5.37 & $0.94(0.83-1.08)$ & 0.39 & & \\
\hline \multicolumn{9}{|c|}{ Housing: eaves of the child's sleeping room } \\
\hline Open & 138 & 299 & 56.57 & 5.29 & - & & & \\
\hline Closed & 102 & 229 & 41.60 & 5.51 & $1.04(0.91-1.20)$ & 0.57 & & \\
\hline \multicolumn{9}{|c|}{ Cows, horses, sheep or goats within $5 \mathrm{~m}$ of child's home } \\
\hline Present & 169 & 371 & 67.80 & 5.47 & - & & & \\
\hline Absent & 80 & 174 & 33.51 & 5.19 & $0.95(0.83-1.09)$ & 0.46 & & \\
\hline \multicolumn{9}{|c|}{ Euclidean distance to the nearest positive aquatic habitat } \\
\hline$<=300 \mathrm{~m}$ & 127 & 266 & 51.76 & 5.14 & - & - & & \\
\hline$>300 \mathrm{~m}$ & 125 & 284 & 50.72 & 5.60 & $1.09(1.00-1.19)$ & 0.06 & & \\
\hline \multicolumn{9}{|c|}{ Distance by road to nearest health centre } \\
\hline$<=2 \mathrm{~km}$ & 119 & 252 & 49.65 & 5.08 & - & - & & \\
\hline$>2 \mathrm{~km}$ & 133 & 298 & 52.83 & 5.64 & $1.11(1.00-1.24)$ & 0.06 & & \\
\hline \multicolumn{9}{|l|}{ EIR (village-level) } \\
\hline- & - & - & - & & $1.00(1.00-1.00)$ & 0.08 & & \\
\hline \multicolumn{9}{|c|}{$\begin{array}{l}\text { \% mortality in WHO tube test with } 0.05 \% \\
\text { deltamethrin diagnostic dose }\end{array}$} \\
\hline- & - & - & - & - & $1.05(0.73-1.50)$ & 0.79 & & \\
\hline
\end{tabular}

IRR incidence rate ratio, *IRR adjusted for travel history, $\$$ IRR adjusted for SES factor score

the most poor children [39]. The same pattern was also observed when SES quintiles were included in the model. The SES factor score was used in the multivariable model instead of the study quintiles since, unlike quintiles which allocate children into categories, the factor score better reflects the range of values in the dataset. The finding that the least poor children have a higher $P$. falciparum infection risk is not explained by a greater number of passively detected infections in these children. Passive cases contributed only $4 \%$ of all cases identified and there were no differences in this proportion across wealth quintiles. Plasmodium falciparum infection rates may be higher in the least poor children due to lower ITN use than the most poor children. Only $53.2 \%$ of the least poor children were reported to use an ITN the previous night compared to over $82.6 \%$ of children in the lowest quintile (most poor).

No association was found between P. falciparum infection risk and either of the two entomological variables hypothesized to impact on the primary outcome, namely EIR and insecticide resistance. The lack of association with EIR and vector density is counter-intuitive given it has been demonstrated in several other studies [40, 41], but could arise if vector densities in all villages were sufficiently high to maintain high transmission, or due to outdoor biting. Although levels of insecticide resistance varied between villages, the rate of $P$. falciparum infection was not greater in villages with higher levels of resistance. This finding may be partly due to the relatively small number of villages surveyed for insecticide resistance, and the resulting lack of statistical power, although no evidence of an association between malaria and insecticide resistance has been found in large area studies in the Sudan and Kenya [14]. Despite the high levels of pyrethroid resistance in the study area, no malaria vectors were found under the children's ITN after 1,156 searches. This would imply that the nets are still providing some level of personal protection since a study in The Gambia found malaria mosquitoes under $48.3 \%$ of untreated bed nets following 1584 net searches [42]. Another study conducted in Kenya in an area of pyrethroid resistance found live An. gambiae s.l. in holed ITNs, with significantly higher numbers in nets with holes greater than 50 $\mathrm{sq} \mathrm{cm}$ in size [43]. Although not measured in this cohort, 
early evening malaria vector biting is found in the study area (Sanou, pers. commun.). This is a time when it is common to find communities active in the peridomestic environment, for example cooking, eating or socializing. In the study site an estimated $85 \%$ of exposure to malaria vector bites can be prevented by use of ITNs from 22.00 to 05.00 but early evening biting outdoors and sporozoite rates of 3.3\%, mean that residents are still exposed to $\sim 32$ infectious bites per person per year even with high ITN compliance (Sanou, pers. commun.). Early evening biting outdoors when people are active in the peridomestic environment is common across Africa [44], and highlights the need for vector control tools that can protect outdoors, such as insecticide-treated 'eave ribbons', attractive targeted sugar baits or larval source management [45-47].

ITN use was high with $80.6 \%$ of caregivers reporting that the study child slept under an ITN the previous night at the baseline survey. This is in line with other surveys including the 2017-8 MIS survey, which reported that $76.0 \%$ children under 5 years slept under an ITN the previous night [7]. The finding of lower reported ITN use in the least poor children (quintile 5) was unexpected. Other studies indicate higher ITN use among the least poor, including the 2018 Burkina Faso MIS survey in all ages which shows the least poor (quintile 5) have 1.4-1.6 times the odds of using an ITN the previous night compared to the most poor (quintile 1). It will be important to explore the reasons for the apparent lower ITN use among the least poor children in future studies. Data on ITN ownership or access was not collected as part of this study and so it is not known whether this was the cause of lower ITN use among the least poor children. Studies across SSA indicate that the net-use gap is primarily driven by intra-household access [48]. Despite the overall high reported bed net use in the study population, and considering that the NMCP campaign only took place the year before the study started, there was cause for concern about the durability of the ITNs since only $62.2 \%$ of the children's ITNs were in good condition at the final survey. Although a longitudinal survey of the ITNs was not conducted, the finding is supported by studies from other countries. For example, in Tanzania a randomized double-blind prospective evaluation of the lifespan of three ITN products found that the functional survival (ITNs in serviceable condition) was 2 years for Olyset ${ }^{\mathrm{TM}}$ and 2.5 years for Permanet ${ }^{\circledR}$ ITNs [16], the two types of ITN delivered by the Burkina Faso NMCP. Bio-efficacy of the sample of children's ITNs evaluated showed low 24-h mortality of $42.2 \%$ for Olyset ${ }^{\mathrm{TM}}$ nets and $39.9 \%$ for Permanet ${ }^{\circledR}$, substantially below the $\geq 80 \%$ mortality threshold set by WHO. Thus, there is a great deal of uncertainty about net use and the protective efficacy of ITNs in
Burkina Faso. The analysis shows that there was no difference in malaria risk between ITN users and non-users, nor that children sleeping under badly torn nets were more likely to have $P$. falciparum infections than those that slept under good nets.

The study has several limitations. Firstly, and most importantly, the study was probably underpowered to detect small risk factors. The sample size calculation assumed $50 \%$ prevalence of risk factors in the study population, while the study children were, in reality, relatively homogeneous with regard to risk factors shown to be important in other studies, for example, house construction. Secondly, while caregivers reported high compliance with ITN use, assessing ITN use is notoriously difficult and this may have impacted on the ability to identify this as an important risk factor. Indeed, social desirability bias and other forms of error mean that surveys are likely to substantially overestimate use [49], and objective and unobtrusive tools to measure ITN compliance are not currently available.

Burkina Faso is one of ten SSA countries designated as a High Burden High Impact country with a response plan including increased political will, strategic use of data to deploy tools for maximum impact and a multi-sectoral approach. While this study generates useful data on malaria burden in Banfora District, it is clear that additional tools will be needed to reduce this burden. Dualactive ITNs (pyrethroid plus piperonyl butoxide (PBO) or pyrethroid plus chlofenapyr) are now being deployed in the study area. PBO-pyrethroid ITNs have been shown to be more effective in reducing malaria than pyrethroid-only ITNs in areas of pyrethroid-resistant vectors [50,51], and monitoring of the effectiveness of these dual active ITNs is ongoing in Burkina Faso. IRS should also be considered as it has been shown to be effective in reducing malaria in other high-burden countries. In an area of Uganda with an EIR of 176 infective bites/ year, three rounds of IRS with the carbamate insecticide, bendiocarb, every six months reduced malaria incidence from 3.3 episodes to 0.6 episodes per person year [52]. Even this effective combination of interventions is, however, insufficient to eliminate malaria, so further interventions are required. SMC is currently being used in 12 sub-Sahelian countries, including Burkina Faso, in children up to 5 years of age, with a protective efficacy of over $50 \%$ against parasitaemia in Burkina Faso [22, 53]. A recent trial in Senegal found similarly high reductions in malaria when SMC was used in children aged under 5 years and in children aged 5 to 9 years [54]. Expanding the age range eligible for SMC could, therefore, be effective in reducing malaria further in Burkina Faso. Burkina Faso is also the site of pilot testing of gene drive mosquitoes, which if proven to be efficacious, feasible and 
acceptable, could be a potential option for malaria control in Burkina Faso.

\section{Conclusions}

The study found overwhelmingly high levels of malaria transmission in Banfora district, in southwest Burkina Faso, and the risk factor survey did not identify any risk factors for further investigation to reduce the malaria burden. The findings have implications for achievement of the ambitious goals set out in the WHO Global Technical Strategy [55]. Malaria elimination in this area of intense seasonal transmission can only be achieved through the use of additional interventions.

\section{Abbreviations}

ACT: Artemisinin combination therapy; AL: Artemether-lumefantrine; Cl: Confidence interval; CNRFP: Centre National de Recherche et de Formation sur le Paludisme; EIR: Entomological inoculation rate; IRR: Incidence rate ratio; IRS: Indoor residual spraying; ITN: Insecticide-treated net; NMCP: National Malaria Control Programme; PBO: Piperonyl butoxide; PCR: Polymerase chain reaction; pHI: Proportionate hole index; SD: Standard deviation; SES: Socio-economic status; SMC: Seasonal malaria chemoprevention; SSA: Sub-Saharan Africa; WHO: World Health Organization.

\section{Acknowledgements}

Authors wish to thank the CNRFP staff, community members, opinion leaders, the community health workers, research assistants, field supervisors and workers whose cooperation and help made this study possible. Special thanks to Issiaka Soulama for supervising PCR analysis in CNRFP, Blami Kote for contribution to data analysis and Luca Nelli for providing the distances to the nearest health centre. Thank you very much to Sodiomon B Sirima for all the support during this work.

The results of this study were presented at the American Society of Tropical Medicine and Hygiene Annual Meeting in 2019, National Harbor, USA.

\section{Authors' contributions}

Conceived and designed the study: SWL, ALW, ABT, NFS, HR, EW. Conducted field and laboratory work: JBY, ABT, AO, AD, ML, KHT, AS, WMG. Conducted data analysis: JBY, ALW, SWL, ABT, ZAO, AO, EA, EW. Contributed to and approved the final manuscript: JBY, SWL, ALW, ABT, NFS, HR, AO, AD, ML, KHT, AS, WMG, EA, EW. All authors read and approved the final manuscript.

\section{Funding}

This project was supported by the Wellcome Trust (Wellcome Trust Collaborative Award "Improving the efficacy of malaria prevention in an insecticide resistant Africa (MiRA)" to the Liverpool School of Tropical Medicine grant agreement number 200222/Z/15/Z). The funder had no role in study design, data collection and analysis, decision to publish, or preparation of the manuscript.

\section{Availability of data and materials}

The datasets used and/or analysed during the current study are available from the corresponding author on reasonable request.

\section{Ethics approval and consent to participate}

The caregivers of study participants provided informed consent (or assent of child if aged 12-15 years) to participate in the study. Study documents were approved by the Burkina Faso Ministry of Health Research Ethics Committee (Deliberation No 2016-12-137), CNRFP Institutional Bioethics Committee (N²016/000007/MS/SG/CNRFP/CIB), Durham University Department of Biosciences Ethics Committee (SBBS/EC/MIRA) and Liverpool School of Tropical Medicine Ethical Committee (Protocol number: 16/047). The study was conducted in compliance with principles set out by the International Conference on Harmonization Good Clinical Practice, the Declaration of Helsinki and the regulatory requirements of Burkina Faso.

\section{Consent for publication}

Not applicable.

\section{Competing interests}

The authors declare that they have no competing interests. All authors declare that they had full access to all of the data in the study and take responsibility for the integrity of the data and the accuracy of the data analysis.

\section{Author details \\ ${ }^{1}$ Centre National de Recherche et de Formation sur le Paludisme, Ouagadou- gou, Burkina Faso. ${ }^{2}$ Department of Biosciences, Durham University, Durham, UK. ${ }^{3}$ Department of Vector Biology, Liverpool School of Tropical Medicine, Liv- erpool, UK. ${ }^{4}$ Institute of Biodiversity Animal Health \& Comparative Medicine, Glasgow University, Glasgow, UK.}

Received: 17 July 2020 Accepted: 7 October 2020

Published online: 16 October 2020

\section{References}

1. WHO. World Malaria Report 2019. Geneva: World Health Organization; 2019.

2. Bhatt S, Weiss DJ, Cameron E, Bisanzio D, Mappin B, Dalrymple U, et al. The effect of malaria control on Plasmodium falciparum in Africa between 2000 and 2015. Nature. 2015;526:207-11.

3. WHO. RBM Partnership to End Malaria High burden to high impact: a targeted malaria response. Geneva: World Health Organization; 2019.

4. Ministère de la Santé Burkina Faso. Annuaire statistique 2018. Ouagadougou: Ministère de la Santé Burkina Faso; 2019.

5. Ministère de la Santé Burkina Faso. Annuaire statistique 2016. Ouagadougou: Ministère de la Santé Burkina Faso; 2017.

6. Ministère de la Santé Burkina Faso. Annuaire statistique 2017. Ouagadougou: Ministère de la Santé Burkina Faso; 2018.

7. Louis VR, Schoeps A, Tiendrebéogo J, Beiersmann C, Yé M, Damiba MR, et al. An insecticide-treated bed-net campaign and childhood malaria in Burkina Faso. Bull World Health Organ. 2015;93:750-8.

8. Namountougou M, Simard F, Baldet T, Diabaté A, Ouédraogo JB, Martin T, et al. Multiple insecticide resistance in Anopheles gambiae s.l. populations from Burkina Faso West Africa. PLoS ONE. 2012;7:e48412.

9. Toé KH, Jones CM, N'Fale S, Ismail HM, Dabiré RK, Ranson H. Increased pyrethroid resistance in malaria vectors and decreased bed net effectiveness in Burkina Faso. Emerg Infect Dis. 2014;20:1691-6.

10. Hughes A, Lissenden N, Viana M, Toé KH, Ranson H. Anopheles gambiae populations from Burkina Faso show minimal delayed mortality after exposure to insecticide-treated nets. Parasit Vectors. 2020;13:17.

11. Churcher TS, Lissenden N, Griffin JT, Worrall E, Ranson H. The impact of pyrethroid resistance on the efficacy and effectiveness of bednets for malaria control in Africa. Elife. 2016;4:e16090.

12. Pryce J, Richardson $M$, Lengeler C. Insecticide-treated nets for preventing malaria. Cochrane Database Syst Rev. 2018;11:CDd000363.

13. Strode C, Donegan S, Garner P, Enayati AA, Hemingway J. The impact of pyrethroid resistance on the efficacy of insecticide-treated bed nets against African anopheline mosquitoes: systematic review and metaanalysis. PLoS Med. 2014;11:e1001619.

14. Kleinschmidt I, Bradley J, Knox TB, Mnzava AP, Kafy HT, Mbogo C, et al. Implications of insecticide resistance for malaria vector control with longlasting insecticidal nets: a WHO-coordinated, prospective, international, observational cohort study. Lancet Infect Dis. 2018;18:640-9.

15. Obi E, Okoh F, Blaufuss S, Olapeju B, Akilah J, Okoko OO, et al. Monitoring the physical and insecticidal durability of the long-lasting insecticidal net DawaPlus ${ }^{\circledR} 20$ in three States in Nigeria. Malar J. 2020;19:124.

16. Lorenz LM, Bradley J, Yukich J, Massue DJ, Mboma ZM, Pigeon O, et al. Comparative functional survival and equivalent annual cost of three long lasting insecticidal net (LLIN) products in Tanzania: a randomised trial with 3-year follow up. PLoS Med. 2020;17:e1003248.

17. Hakizimana E, Cyubahiro B, Rukundo A, Kabayiza A, Mutabazi A, Beach R, et al. Monitoring long-lasting insecticidal net (LLIN) durability to validate net serviceable life assumptions in Rwanda. Malar J. 2014;13:344. 
18. Bhatt S, Weiss DJ, Mappin B, Dalrymple U, Cameron E, Bisanzio D, et al. Coverage and system efficiencies of insecticide-treated nets in Africa from 2000 to 2017. Elife. 2015;4:e09672.

19. Tusting LS, Ippolito MM, Willey BA, Kleinschmidt I, Dorsey G, Gosling RD, et al. The evidence for improving housing to reduce malaria: a systematic review and meta-analysis. Malar J. 2015;14:e209.

20. Tiono AB, Kangoye DT, Rehman AM, Kargougou DG, Kabore Y, Diarra A, et al. Malaria incidence in children in South-West Burkina Faso: comparison of active and passive case detection methods. PLoS ONE. 2014;9:e86936.

21. Tiono AB, Guelbeogo MW, Sagnon NF, Nebie I, Sirima SB, Mukhopadhyay A, et al. Dynamics of malaria transmission and susceptibility to clinical malaria episodes following treatment of Plasmodium falciparum asymptomatic carriers: results of a cluster-randomized study of communitywide screening and treatment, and a parallel entomology study. BMC Infect Dis. 2013;13:535.

22. WHO. WHO policy recommendation: Seasonal Malaria Chemoprevention (SMC) for Plasmodium falciparum malaria control in highly seasonal transmission areas of the Sahel sub-region in Africa. Geneva: World Health Organization; 2012.

23. Gonçalves BP, Kapulu MC, Sawa P, Guelbéogo WM, Tiono AB, Grignard $L$, et al. Examining the human infectious reservoir for Plasmodium falciparum malaria in areas of differing transmission intensity. Nat Commun. 2017:8:1133.

24. Ministère de la Santé Burkina Faso. Directives nationales pour la prise en charge du paludisme dans les formations sanitaires du Burkina Faso. Ouagadougou: Ministère de la Santé Burkina Faso; 2014.

25. Snounou G, Viriyakosol S, Zhu XP, Jarra W, Pinheiro L. do RV, Thaithong $\mathrm{S}$, Brown KN: High sensitivity of detection of human malaria parasites by the use of nested polymerase chain reaction. Mol Biochem Parasitol. 1993;61:315-20.

26. Institut National de la Statistique et de la Démographie (INSD), Programme National de Lutte contre le Paludisme (PNLP), ICF International. Enquête sur les indicateurs du paludisme (EIPBF). Ouagadougou: INSD, PNLP and ICF International, 2015.

27. WHO. Guidelines for monitoring the durability of long-lasting insecticidal mosquito nets under operational conditions. Geneva: World Health Organization; 2011

28. WHO. Guidance note for estimating the longevity of long-lasting insecticidal nets in malaria control. Geneva: World Health Organization; 2013.

29. WHO. Guidelines for laboratory and field testing of long-lasting insecticidal nets. Geneva: World Health Organization; 2013.

30. Scott JABW, Collins FH. Identification of single specimens of the Anopheles gambiae complex by the polymerase chain reaction. Am J Trop Med Hyg. 1993;4:520-9.

31. WHO. Test procedures for insecticide resistance monitoring in malaria vector mosquitoes -. 2nd ed. Geneva: World Health Organization; 2016.

32. Kirkwood BR, Sterne JAC. Essential Medical Statistics. 2nd ed. Hoboken: Blackwell Science Ltd; 2003.

33. von Elm E, Altman DG, Egger M, Pocock SJ, Gøtzsche PC, Vandenbroucke JP, STROBE Initiative. The strengthening the reporting of observational studies in epidemiology (STROBE) statement: guidelines for reporting observational studies. Lancet. 2007:370:1453-7.

34. Diallo A, Sié A, Sirima S, Sylla K, Ndiaye M, Bountogo M, et al. An epidemiological study to assess Plasmodium falciparum parasite prevalence and malaria control measures in Burkina Faso and Senegal. Malar J. 2017;16:63.

35. Wehner S, Stieglbauer G, Traore C, Sie A, Becher H, Muller O. Malaria incidence during early childhood in rural Burkina Faso: analysis of a birth cohort protected with insecticide-treated mosquito nets. Acta Trop. 2017;175:78-83.

36. Ouédraogo A, Tiono AB, Diarra A, Sanon S, Yaro JB, Ouedraogo E, et al. Malaria morbidity in high and seasonal malaria transmission area of Burkina Faso. PLoS ONE. 2013;8:e50036.

37. Smith C, Whittaker M. Beyond mobile populations: a critical review of the literature on malaria and population mobility and suggestions for future directions. Malar J. 2014;13:307.

38. Arinaitwe E, Dorsey G, Nankabirwa Jl, Kigozi SP, Katureebe A, Kakande $\mathrm{E}$, et al. Association between recent overnight travel and risk of malaria: a prospective cohort study at 3 sites in Uganda. Clin Infect Dis. 2019;68:313-20.

39. Tusting LS, Willey B, Lucas H, Thompson J, Kafy HT, Smith R, et al. Socioeconomic development as an intervention against malaria: a systematic review and meta-analysis. Lancet. 2013;382:963-72.

40. Kirby MJ, Ameh D, Bottomley C, Green C, Jawara M, Milligan PJ, et al. Effect of two different house screening interventions on exposure to malaria vectors and on anaemia in children in The Gambia: a randomised controlled trial. Lancet. 2009;374:998-1009.

41. Adiamah JH, Koram KA, Thomson MC, Lindsay SW, Todd J, Greenwood BM. Entomological risk factors for severe malaria in a peri-urban area of The Gambia. Ann Trop Med Parasit. 1993:87:491-500.

42. Lindsay SW, Alonso PL, Armstrong Schellenberg JR, Hemingway J, Adiamah JH, Shenton FC, et al. A malaria control trial using insecticide-treated bed nets and targeted chemoprophylaxis in a rural area of The Gambia, West Africa. 7. Impact of permethrin-impregnated bed nets on malaria vectors. Trans R Soc Trop Med Hyg. 1993;87:45-51.

43. Ochomo EO, Bayoh NM, Walker ED, Abongo BO, Ombok MO, Ouma C, et al. The efficacy of long-lasting nets with declining physical integrity may be compromised in areas with high levels of pyrethroid resistance. Malar J. 2013;12:368.

44. Sherrard-Smith E, Skarp J, Beale A, Fornadel C, Norris L, Moore SJ, et al. Mosquito feeding behaviour and how it influences residual malaria transmission across Africa. Proc Natl Acad Sci USA. 2019;116:15086-95.

45. Choi L, Majambere S, Wilson AL. Larviciding to prevent malaria transmission. Cochrane Database Syst Rev. 2019;8:CD012736.

46. Mmbando AS, Ngowo H, Limwagu A, Kilalangongono M, Kifungo K, Okumu FO. Eave ribbons treated with the spatial repellent, transfluthrin, can effectively protect against indoor-biting and outdoor-biting malaria mosquitoes. Malar J. 2018;17:368.

47. Traore MM, Junnila A, Traore SF, Doumbia S, Revay EE, Kravchenko VD, et al. Large-scale field trial of attractive toxic sugar baits (ATSB) for the control of malaria vector mosquitoes in Mali. West Africa Malar J. 2020;19:72.

48. Koenker H, Kilian A. Recalculating the net use gap: a multi-country comparison of ITN use versus ITN access. PLoS ONE. 2014;9:e97496.

49. Krezanoski PJ, Bangsberg DR, Tsai AC. Quantifying bias in measuring insecticide-treated bednet use: meta-analysis of self-reported vs objectively measured adherence. J Glob Health. 2018;8:010411.

50. Protopopoff N, Mosha JF, Lukole E, Charlwood JD, Wright A, Mwalimu $C D$, et al. Effectiveness of a long-lasting piperonyl butoxide-treated insecticidal net and indoor residual spray interventions, separately and together, against malaria transmitted by pyrethroid-resistant mosquitoes: a cluster, randomised controlled, two-by-two factorial design trial. Lancet. 2018;391:1577-88.

51. Staedke SG, Gonahasa S, Dorsey G, Kamya MR, Maiteki-Sebuguzi C, Lynd $A$, et al. Effect of long-lasting insecticidal nets with and without piperonyl butoxide on malaria indicators in Uganda (LLINEUP): a pragmatic, clusterrandomised trial embedded in a national LLIN distribution campaign. Lancet. 2020;395:1292-303.

52. Katureebe A, Zinszer K, Arinaitwe E, Rek J, Kakande E, Charland K, et al. Measures of malaria burden after long-lasting insecticidal net distribution and indoor residual spraying at three sites in Uganda: a prospective observational study. PLoS Med. 2016;13:e1002167.

53. Druetz T, Corneau-Tremblay N, Millogo T, Kouanda S, Ly A, Bicaba A, et al. Impact evaluation of seasonal malaria chemoprevention under routine program implementation: a quasi-experimental study in Burkina Faso. Am J Trop Med Hyg. 2018;98:524-33.

54. Ndiaye JLA, Ndiaye Y, Ba MS, Faye B, Ndiaye M, Seck A, et al. Seasonal malaria chemoprevention combined with community case management of malaria in children under 10 years of age, over 5 months, in south-east Senegal: a cluster-randomised trial. PLoS Med. 2019;16:e1002762.

55. WHO. Global technical strategy for malaria 2016-2030. Geneva: World Health Organization; 2016.

\section{Publisher's Note}

Springer Nature remains neutral with regard to jurisdictional claims in published maps and institutional affiliations. 